\title{
Non-Occlusive Mesenteric Ischemia in a Hemodialysis Patient
}

\author{
Akihito Tanaka, Yukiko Ito, Yasuhiro Sugiura and Ryozo Sezaki
}

Key words: non-occlusive mesenteric ischemia, hemodialysis

(Intern Med 50: 523, 2011)

(DOI: 10.2169/internalmedicine.50.4844)

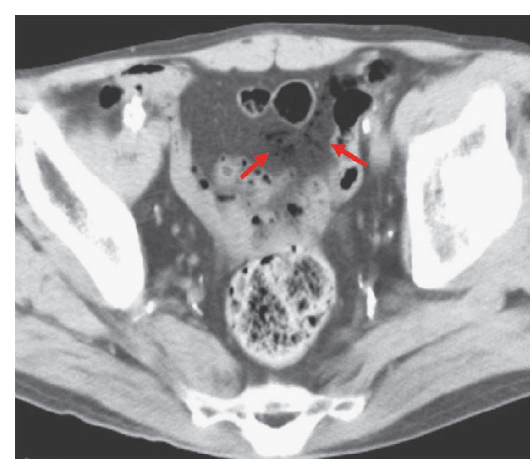

Picture 1.

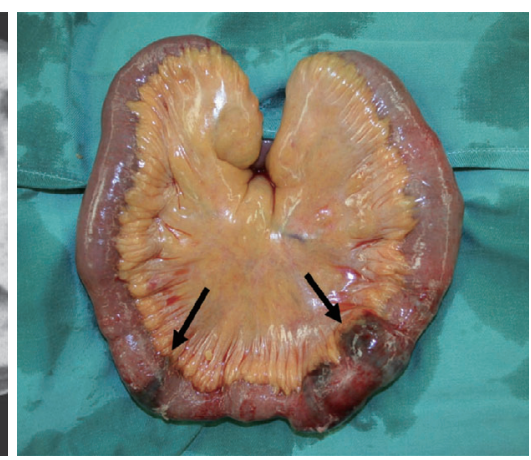

Picture 2.

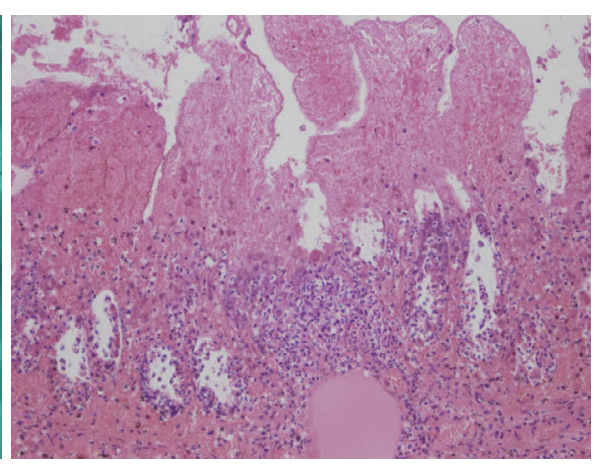

Picture 3.
A 78-year-old man with a history of chronic renal failure due to nephrosclerosis started hemodialysis in 2002. He sometimes complained of abdominal pain with the advance of dehydration on hemodialysis. In October 2007, he complained about abdominal pain after hemodialysis. Abdominal CT demonstrated significant large artery calcification and free air near the colon sigmoideum (Picture 1). Digestive tract perforation was suspected and an emergent operation was performed. Two necrosis spots were found in the distal ileum (Picture 2) and partial resection of the small intestine was performed. His postoperative course was good and he was discharged. Later, pathological examination revealed that there was a ghost-like appearance without thromboembolism (Picture 3). Thus, we considered the possibility of non-occlusive mesenteric ischemia (NOMI).

NOMI is considered as a low perfusion syndrome. Hemodialysis patients often have severe arteriosclerosis and are dehydrated each time. Therefore, hemodialysis patients are a high risk group for NOMI (1). In cases of abdominal pain among hemodialysis patients, ruling out NOMI is important.

The authors state that they have no Conflict of Interest (COI).

\section{Reference}

1. John AS, Tuerff SD, Kerstein MD. Nonocclusive mesenteric infarction in hemodialysis patients. J Am Coll Surg 190: 84-88, 2000 .

Department of Nephrology, Nagoya Ekisaikai Hospital, Japan

Received for publication November 13, 2010; Accepted for publication November 23, 2010

Correspondence to Dr. Akihito Tanaka, zhangren_at_23@yahoo.co.jp

(C) 2011 The Japanese Society of Internal Medicine Journal Website: http://www.naika.or.jp/imindex.html 